\title{
Inflammation et immunité : implications dans l'obésité et le diabète de type 2
}

\author{
Naim Akhtar KHAN \\ Université de Bourgogne, \\ Faculté des sciences de la vie, \\ Département de physiologie animale, \\ UPRES Lipides \& Nutrition, \\ 6, Boulevard Gabriel, \\ 21000 Dijon, France \\ $<$ Naim.Khan@u-bourgogne.fr>
}

\begin{abstract}
The evidences have been increasingly accumulated on the implication of inflammatory mediators like tumor necrosis factor- $\alpha$ (TNF- $\alpha$ ) and interleukin- 6 (IL-6) in the pathological states related to insulin resistance like obesity, type 2 diabetes and atherosclerosis. There seems a link between insulin resistance and these pro-inflammatory agents, secreted by macrophages and adipocytes. Th (helper) cells are differentiated into either Th1 or Th2 phenotypes. It is generally considered that Th1 phenotype is pro-inflammatory whereas Th2 phenotype exerts anti-inflammatory (protective) effects. The upregulation of Th1 phenotype may aggravate these pathologies. One of the adipokines, i.e., adiponectin, and insulin act as anti-inflammatory agents. Insulin also favours the differentiation of Th cells into Th2 phenotype. TNF- $\alpha$ and IL-6 might counter balance the action of insulin by interfering with insulin receptor signalling in these pathological situations. The agonists of PPAR $\alpha$ and PPAR , and $n-3$ polyunsaturated fatty acids may exert an anti-inflammatory effect by shifting Th1/Th2 balance to Th2 phenotype. The factors, implicated in the secretion of inflammatory mediators are not well known, though the role of glucose-induced oxidative stress has been underlined. In this article, we shed light on the cross-talk between pro- and anti-inflammatory agents in these patho-physiological states related to insulin resistance.
\end{abstract}

Key words: obesity, inflammation, immune system, PPAR, fatty acids

\section{Cellules Th1 (pro-inflammatoires) et cellules Th2 (anti-inflammatoires)}

Le système immunitaire est, principalement, composé de cellules - $T$ (responsables de l'immunité à médiation cellulaire), de cellules $B$ (responsables de l'immunité humorale) et de macrophages (responsables de l'inflammation). Les cellules -T peuvent être divisées en deux populations: T-helper $\left(\mathrm{CD}^{+}\right)$et T-cytotoxique $\left(\mathrm{CD}^{+}\right)$. Les cellules Th (helper ou auxiliaire ou Th0) ont la particularité de reconnaître des peptides étrangers issus de la dégradation des protéines internalisées par endocytose et présentées à la surface des cellules phagocytaires par les protéines $\mathrm{CMH}$ de classe II. La stimulation plus ou moins prolongée par un antigène spécifique permet la différenciation des cellules Th0 vers deux phénotypes: Th1 ou Th2. Ces cellules sont caractérisées par des profils de synthèse de cytokines différentes (figure 1). Les cellules Th1 produisent l'interleukine-2 (IL-2) et l'interféron- $\gamma$ $(\mathrm{IFN}-\gamma)$ : elles activent les monocytes et les macrophages et sont donc considérées comme pro-inflammatoires. Par contre, les cellules Th2 produisant I'IL-4, I'IL-5, I'IL-6, I'IL-10 et I'IL-13, sont considérées comme anti-inflammatoires : elles stimulent les éosinophiles et les mastocytes, et augmentent la synthèse d'anticorps, en particulier de classe lgE (immunoglobulines de type E), ce qui supprime l'immunité à médiation cellulaire. Le profil initial de cytokines, dont la synthèse est déclenchée par le patho- gène, et la concentration locale de différents stéroïdes déterminent l'orientation des cellules Th0 vers les deux sous-populations. Par exemple, si un micro-organisme déclenche la sécrétion d'IL-12 par les macrophages et d'IFN- $\gamma$ par les cellules NK (natural killer), la différenciation sera orientée vers le phénotype Th1, tandis que la libération d'IL-4 favorise une réponse de type Th2. Une fois établi, chaque phénotype tend à supprimer le phénotype opposé. En effet, I'IFN- $\gamma$ des cellules Th1 inhibe la prolifération des cellules Th2, alors que I'IL-10 des cellules Th2 bloque la production de cytokines par les Th1 (figure 1).

\section{Cellules Th1 et Th2 dans l'obésité/I'insulino-résistance}

Dans cet article, nous ne décrirons pas le rôle des cellules Th1 dans la pathogenèse du diabète de type 1 car il est bien connu que ces cellules, après l'infiltration du pancréas, détruisent les cellules bêta et aggravent cette pathologie $[4,5]$.

Nous avons récemment développé un modèle de bébés macrosomiques, nés de mères diabétiques, qui à l'age adulte deviennent obèses, marqués par l'augmentation de taux de lipides (cholestérol total, LDL, VLDL, etc.) et qui développent l'insulino-résistance. Nous avons constaté que ces animaux obèses, ont un taux très élevé de cytokines pro-inflammatoires, IFN- $\gamma$ et I'IL-2 [6]. Chez ces animaux, il n'y a pas une modification du taux de I'IL-4, mais le rapport IFN- $\gamma /$ IL-4 augmente de l'ordre de 21 , suggérant que la différenciation de cellules Th0 vers 


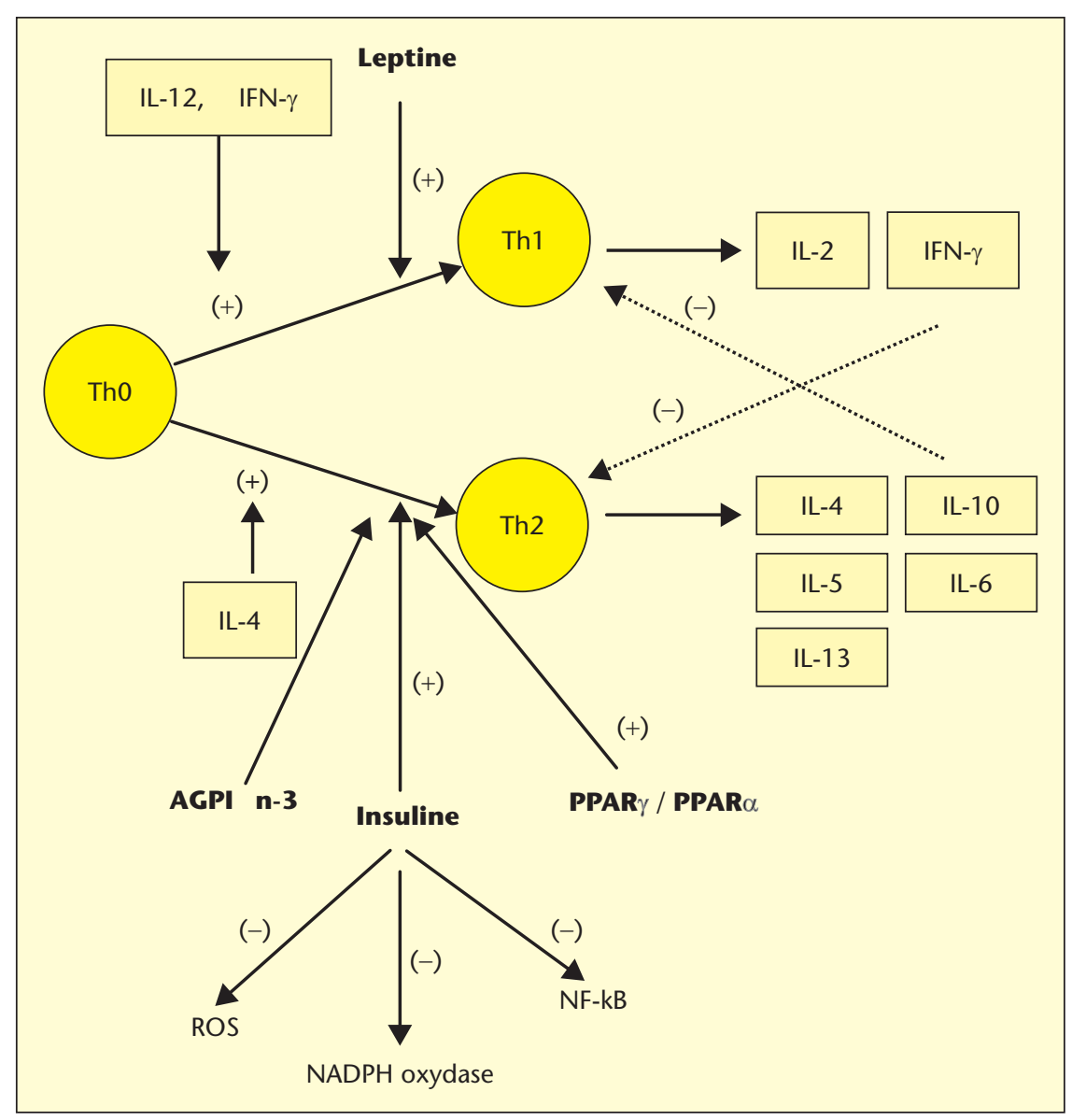

Figure 1. Différenciation des lymphocytes Th0 en cellules Th1 et Th2 et, leurs modulations.

Ces cellules sont identifiées par la sécrétion de leurs cytokines respectives. Les cellules Th0, sécrétant I'IL-2, se différencient en Th1, sous I'action de I'L-12 et I'IFN- $\gamma$, libérés, respectivement, par les macrophages et les cellules NK (natural killer). Par ailleurs, le phénotype Th2 est induit par l'action de I'IL-4, produite par les mastocytes. L'IFN- $\gamma$ et I'IL-10, à leur tour, exercent un effet inhibiteur, respectivement sur la différenciation de phénotypes Th2 et Th1. L'insuline, les agonistes de PPAR et les AGPI n-3 favorisent la différenciation en phénotype Th2. L'insuline diminue aussi la production de ROS et l'activité du NADPH oxydase et du NF-kB. Cependant, la leptine favorise la différenciation en cellules Th1. (+), effet inducteur; (-), effet inhibiteur.

le phénotype Th1 est accélérée lors de l'installation de l'obésité [6]. À notre surprise, nous avons constaté que le taux de cytokines des cellules Th1 diminue chez les sujets atteints du diabète gestationnel [7]. En effet, il a été démontré qu'une baisse du phénotype Th1 est impliquée dans l'installation de la grossesse. Cependant, les cytokines pro-inflammatoires (TNF- $\alpha$ et IL-6) augmentent chez ces sujets. Or, les bébés obèses, nés de ces mères, ont une différenciation accélérée de cellules Th1, indiquant un état inflammatoire de ces enfants. Verwaerde et al. [8] ont effectué une étude sur les souris, maintenues sous le régime riche en lipides. Ils ont démontré que le régime HFD (en anglais, high fat diet) module les fonctions lymphocytaires où on constate l'augmentation du rapport IFN- $\gamma / \mathrm{IL}-4$, indiquant un rôle du système immunitaire dans le micro-environnement hépatique chez la souris ob/ob. Ils ont constaté que le HFD induit l'obésité et oriente la différenciation des cellules T hépatiques vers le phénotype Th1 chez ces animaux. Ainsi, les cellules Th1 étaient responsables de l'inflammation hépatique et pouvaient aggraver l'obésité.

\section{Modulation de cellules Th1/Th2 par la leptine, I'adiponectin et I'insuline}

La leptine, impliquée dans la régulation de I'obésité, en modifiant le métabolisme lipidique, diminue l'appétit en interférant avec le neuropeptide $Y$ et le récepteur de la mélanocortine dans I'hypothalamus $[9,10]$. La leptine est également produite par le placenta. La leptine est élevée chez les sujets obèses et elle exerce un effet agrégeant sur les plaquettes, et par conséquent, elle pourrait réguler le système immunitaire.
Les récepteurs de la leptine ont été récemment identifiés sur les lymphocytes murins [11]. Ces récepteurs inhibent l'apoptose cellulaire $T$ et favorisent la survie des cellules T en modifiant la signalisation via STAT-3. En ce qui concerne la différenciation cellulaire $T$, la leptine induit la polarisation de cellules Th0 vers le phénotype Th1 et augmente la sécrétion de cytokines proinflammatoires. Une étude effectuée sur les enfants obèses indique une corrélation positive entre le pourcentage de cellules $T$ sécrétant I'IFN- $\gamma$, la concentration de leptine et l'insulinorésistance [12]. Lorsque les cellules dendritiques humaines sont traitées par la leptine, on constate que ces cellules favorisent la polarisation de cellules Th0 vers le phénotype Th1 et diminuent la sécrétion de I'IL-10, une cytokine de cellules Th2 [13]. Chez les enfants malnutris, l'augmentation du poids corporel est liée à l'augmentation de taux de la leptine et de la différenciation accélérée de cellules Th1 qui secrètent abondamment I'IFN- $\gamma$ [14]. II est clair que la leptine exerce une action proinflammatoire en ce qui concerne la différenciation de cellules Th0.

En ce qui concerne l'adiponectine, nous ne disposons pas d'étude directe qui démontre son action sur la différenciation de ces cellules, mais il a été démontré que l'inflammation hépatique induite par l'activation des cellules $T$ est associée à une baisse du taux de l'adiponectine [15], suggérant que cette adipokine pourrait exercer un effet anti-inflammatoire.

Les lymphocytes $T$, non-activés, n'expriment pas les récepteurs de l'insuline (IR] mais lors de l'activation lymphocytaire, ces cellules expriment les IR et l'insuline oriente la différenciation de lymphocytes Th0 vers le phénotype Th2 [16], indiquant que les effets antiinflammatoires de cette hormone passeraient également par une action directe sur ces cellules immunitaires. Ces observations, à nouveau, démontrent une interaction entre la différenciation des cellules T et cette hormone hypoglycémiante.

\section{PPAR modulent la différenciation cellulaire Th/Th2}

Les PPAR (en anglais, peroxisome proliferatoractivated receptors) sont facteurs de transcription qui régulent, sous influence d'un ligand, un grand nombre de gènes en modifiant l'activation ou la répression transcriptionnelle [17]. Trois isoformes des PPAR ont été identifiés chez les vertébrés inférieurs et les mammifères [18]. Les PPAR qui sont bien caractérisés, en ce qui concerne leur distribution, leur fonction et leur spécificité, sont PPAR $\alpha, \operatorname{PPAR} \beta(\delta)$ et PPAR $\gamma$ [19]. Dans cet article, nous n'allons pas décrire, en détail, le rôle de PPAR dans le métabolisme 
lipidique/adipocytaire, mais nous allons citer seulement des études sur leur implication dans I'inflammation/différenciation lymphocytaire.

En plus des adipocytes et le foie, le PPAR $\alpha$ est également exprimé par les cellules de la lignée monocyte/macrophages. Ces cellules expriment également le PPAR $\gamma$, ce qui indique leur rôle dans la régulation du système immunitaire [20-22]. Plusieurs études ont identifié l'expression du PPAR a sur les cellules $T$ et $B$ et son expression semble diminuer lors de l'activation lymphocytaire [20, 23]. Les ligands du PPAR $\alpha$ régulent l'inflammation car ils influencent la différenciation de cellules Th1 [24]. Ces ligands augmentent également l'expression de I'IL-4, une cytokine des cellules Th2 [25]. La plupart des résultats suggèrent que le PPAR $\alpha$ jouerait un rôle immunosuppresseur et favoriserait l'immunité Th2. Maruyama et al. [26] ont démontré que le fénofibrate, un ligand du PPAR $\alpha$, augmente l'expression in vivo de l'IL-10 chez le rat atteint de myocardite auto-immune. D'autres chercheurs ont démontré que les agonistes des PPAR $\alpha$ tels que WY14,643 diminuent la production de I'IFN- $\gamma$ [25]. Le gemfibrozil et le fénofibrate diminuent la libération de I'IFN- $\gamma$ et augmentent celle de I'IL4 [21]. II semblerait qu'il existe une interaction entre la voix de signalisation des PPAR et les facteurs de transcription tels que les STAT et GATA [27]. II est à souligner que lors de la différenciation des cellules Th0 en Th2, le STAT-6 est transloqué vers le noyau et aboutit à l'expression du CATA-3, un régulateur de la différenciation du phénotype Th2 [28]. Cependant, nous ne disposons d'aucune étude sur l'expression du GATA-3 en présence du PPAR $a$ lors de la différenciation lymphocytaire.

Nous avons récemment étudié le rôle du PPARo dans la différenciation Th1/Th2 dans un modèle de l'insulino-résistance [29]. Ainsi, nous avons démontré, dans un premier temps, que la présence de PPAR $\alpha$ est indispensable à la survie des bébés nés de mères diabétiques. De plus, nous avons constaté que pendant le diabète gestationnel chez la souris PPARa-KO, la différenciation des cellules Th1/Th2 est orientée vers le phénotype Th1. Cependant, chez les souris sauvages, cette différenciation est orientée vers le phénotype Th2. De plus, les souris PPARa-KO ont une expression diminuée du phénotype Th2. Ceci démontre que le PPARo est impliqué dans la différenciation de ces cellules vers un phénotype protecteur, Th2.

Le PPAR y est un facteur clé contrôlant la masse corporelle ainsi que la sensibilité à l'insuline. De nombreuses évidences suggèrent l'implication de PPAR $\gamma$ dans I'homéostasie du glucose, le métabolisme des lipides et la sensibilité à l'insuline observée lors du diabète gestationnel [30]. À ce jour, les ligands endogènes de PPAR $\gamma$ incluent différents acides gras et leurs métabo- lites [31]. PPAR $\gamma$ joue un rôle critique dans la différenciation adipocytaire et le stockage des acides gras [32]. La signalisation aberrante de la voie PPARy est associée à une modification de la masse corporelle et de la sensibilité à I'insuline. De plus, le PPARy joue un rôle important dans l'activation des cellules $T$ chez les sujets diabétiques et obèses [33]. En effet, I'activation de PPAR $\gamma$ oriente la balance des cytokines Th1/Th2 vers le phénotype Th2. PPAR $\gamma$ existe sous deux isoformes: PPAR $\gamma 1$ n'est pas restreint aux tissus adipeux, on le retrouve dans d'autres tissus et cellules, notamment les cellules du système immunitaire (macrophages et lymphocytes); PPAR 22 est exprimé uniquement dans les adipocytes [34]. L'activation de PPAR $\gamma$ par les TZD (thiazolidinediones) exerce des propriétés antiinflammatoires [35]. Les activateurs de PPAR $\gamma$ favorisent la réponse Th2 en diminuant la production de I'IFN $\gamma$ par les cellules -T périphériques.

Les macrophages expriment aussi PPAR $\gamma$. Les TZD ainsi que d'autres agonistes de PPAR diminuent la production de TNF- $\alpha$, de l'IL- 6 , du NO, de IL-1 $\beta$, induites par le LPS et I'IFN- $\gamma$ [36]. Ainsi, les TZD, en agissant sur les macrophages/ adipocytes, pourraient diminuer la production des cytokines pro-inflammatoires et par conséquent, exercer des effets bénéfiques sur le métabolisme du glucose et lipidique. Cependant, certains chercheurs ont constaté que les agonistes de PPAR $\gamma$ ne modifient pas la sécrétion de cytokines pro-inflammatoires [37].

\section{TNF- $\alpha$ et IL-6, des agents pro- inflammatoires, dans I'obésité/insulino-résistance}

II a été démontré que le tissu adipeux, mis à part la sécrétion des adipokines (adiponectine et leptine), sécrète également des cytokines pro-inflammatoires, principalement le TNF- $\alpha$ et I'IL-6 (figure 2). Creely et al. [38] ont étudié I'interaction entre les adipokines et les TLR (en anglais, toll-like receptors) sur les adipocytes isolés. Ils en ont conclu que le lipopolysaccharide augmente non seulement la sécrétion de I'IL-6, du TNF- $\alpha$ mais également l'expression de TLR-2 sur les adipocytes via la cascade de I'inflammation.

En ce qui concerne le TNF- $\alpha$, on peut noter les observations suivantes: 1) le TNF- $\alpha$ est exprimé d'une manière constitutive par le tissu adipeux ; 2) les souris génétiquement obèses (ob/ob) et les rats (fa/fa Zucker) expriment abondamment cette cytokine dans leur tissu adipeux $[39,40]$, et 3 ) le TNF- $\alpha$ est le médiateur de l'insulino-résistance chez ces animaux. Le tissu adipeux de sujets obèses contient beaucoup plus de TNF- $\alpha$ que les sujets minces $[41,42]$. Bien que les adipocytes humains expriment le TNF- $\alpha$, comme c'est le cas des macrophages, ils ne répondent pas à l'endotoxine $[39,43]$.

II est à signaler que le tissu adipeux contient non seulement les adipocytes mais aussi les cellules endothéliales et les macrophages. Weisberg et al. [44] ont constaté que les macrophages infiltrent des tissus adipeux chez les sujets obèses. Ils ont démontré que l'indice de masse corporelle est directement lié au degré de l'infiltration de macrophages, exprimant le CD68, d'origine de moelle osseuse. De plus, les adipocytes secrètent le MCP-1 (en anglais, monocyte chemotactic protein-1), un agent chimiotactique monocytaire, et le CSF-1 (en anglais, colony stimulating factor), un facteur responsable de la différenciation monocytemacrophage. Ainsi, on peut dire que les adipocytes libèrent les facteurs qui favorisent l'infiltration et la différenciation des macrophages. Récemment, Lacasa et al. [45] ont suggéré que les macrophages, infiltrés dans le tissu adipeux, pourraient exercer une action paracrine et, par conséquent, moduler les fonctions adipocytaires. Ils ont effectué des expériences sur les adipocytes humains en présence de surnageant de macrophages circulant et ils en ont conclu que le TNF- $\alpha$ monocytaire diminue la différenciation adipocytaire.

II existe une corrélation positive entre le TNF- $\alpha$ et le taux de C-peptide [46]. Le tissu adipeux de sujets obèses contient beaucoup plus de iNOS (en anglais, inducible NO synthase), de TGF- $\beta 1$, de C-réactive protéine et de I'ICAM que les sujets minces [41, 42]. II est intéressant de noter que le taux des ARNm du TNF- $\alpha$ diminue après une baisse de poids chez les sujets obèses $[39,43]$. Clement et al. [47] ont constaté, chez les sujets obèses, qu'un régime peu calorique diminue non seulement la masse graisseuse mais également l'expression des gènes de cytokines pro-inflammatoires dans des adipocytes. Ce régime augmente aussi l'expression des gènes, codant pour les agents antiinflammatoires. D'autres études démontrent aussi qu'une baisse de l'indice de masse corporelle est associée à la diminution de facteurs inflammatoires chez les sujets obèses [48, 49]. Ces observations suggèrent qu'il existe une corrélation directe entre l'obésité et l'état inflammatoire de ces sujets $[1,2,50]$.

Le TNF- $\alpha$ interfère avec le mécanisme d'action de I'insuline, probablement, au niveau de l'activation de tyrosine kinase des récepteurs de I'insuline [51]. L'effet inhibiteur du TNF- $\alpha$ sur l'action de l'insuline va également influencer la lipogenèse adipocytaire et stimuler la lipolyse [52], suggérant que le TNF- $\alpha$ est un modulateur des fonctions adipocytaires. L'administration des récepteurs du TNF- $\alpha$ qui se fixent sur le TNF- $\alpha$ endogène normalise l'insulinosensibilité [53]. Ainsi, on peut conclure que le 


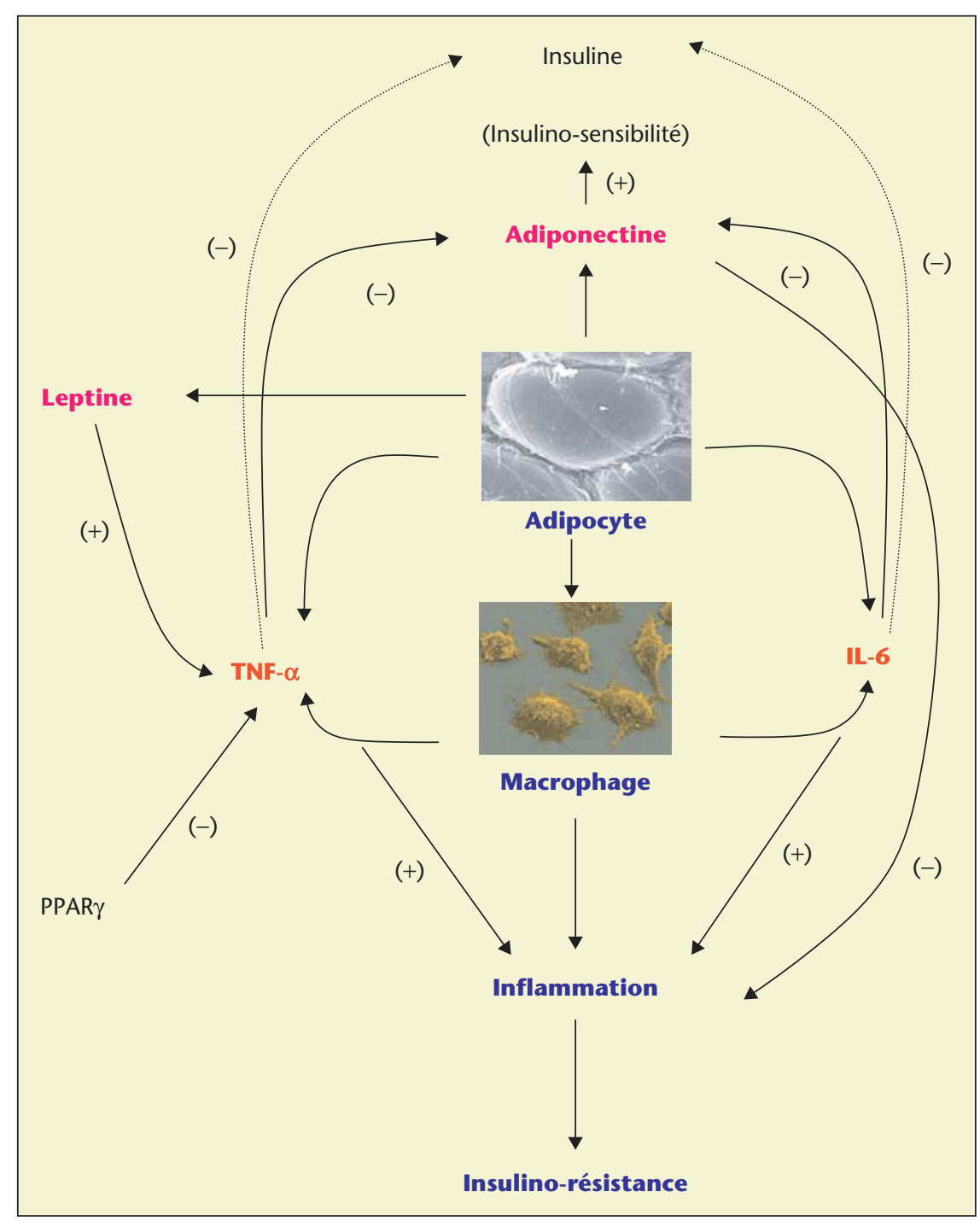

Figure 2. Sécrétion des cytokines et des adipokines, et leurs implications dans l'insulino-résistance. Les adipocytes secrètent les adipokines (adiponectine et leptine) et les cytokines pro-inflammatoires, libérées aussi pas les macrophages, qui favorisent l'inflammation. La leptine contribue à l'inflammation en augmentant la sécrétion du TNF- $\alpha$. Cependant, le PPARy diminue le taux de cette cytokine pro-inflammatoire. Le TNF- $\alpha$ et I'L-6 antagonisent avec l'action de l'insuline et diminuent la sécrétion de l'adiponectine qui a une action insulino-sensibilisante. (+), effet inducteur ; (-), effet inhibiteur.

TNF- $\alpha$ serait responsable de l'insulinorésistance $[1,2]$. En effet, le rôle principal du TNF- $\alpha$ adipocytaire serait d'induire l'inflammation et de prolonger un état d'insulinorésistance.

Il faut rappeler qu'une croissance soutenue de tissu adipeux aboutit au développement de syndrome métabolique marqué par le diabète de type 2, l'hypertension et de l'état inflammatoire. L'inhibiteur d'activateur de plasminogène (PAl-1) constitue l'un des facteurs impliqués dans ce processus. En effet, il a été suggéré que le TNF- $\alpha$ sécrété par les adipocytes est responsable de l'augmentation de la concentration plasmatique de PAI-1, puis de complications cardio-vasculaires [54].
L'IL-6 semble également impliqué dans la pathogenèse du diabète de type 2 , adiposité anormale ou bien les anomalies lipidiques [55]. II est intéressant de noter qu'entre 10 et $30 \%$ de l'IL-6 circulant est dérivé des tissus adipeux, ce qui suggère son rôle probable dans les pathologies métaboliques. L'IL-6 régule aussi l'équilibre énergétique. II existe une corrélation positive entre le taux de I'IL- 6 et de la leptine chez I'homme et I'animal. L'IL-6 peut jouer un rôle dans la signalisation vers le cerveau en ce qui concerne l'adiposité. En effet, l'hypothalamus ventro-médiane, la région qui contrôle l'équilibre énergétique, exprime les récepteurs de I'IL-6 [56]. Les souris, n'exprimant pas le gène de l'IL-6, développent l'obésité prématu- rément car ils mangent la nourriture en excès [57]. L'administration intra-cérébroventriculaire de l'IL-6 corrige l'obésité chez ces animaux [57]. Chez les primates, I'IL-6 exogène diminue la masse graisse [58]. Malgré ces observations, le rôle exact de l'IL-6 dans la régulation de l'obésité $n$ 'est pas bien défini. II existe une corrélation positive entre le taux de I'IL-6 circulant, I'adiposité [55] et l'insulinorésistance [59]. En effet, les sujets atteints du diabète de type 2 ont un taux élevé de I'IL-6 dans leur circulation de sang $[60,61]$.

II a été suggéré que l'augmentation du TNF- $\alpha$ et de l'IL-6 chez les sujets diabétiques est une conséquence du stress oxydatif, induite par I'hyperglycémie [62]. Mohanty et al. [63] ont démontré que l'ingestion du glucose chez les sujets normaux diminuent la concentration de $\alpha$-tocopherol et augmentent l'expression de p47phox dans les cellules mononucléaires périphériques. De plus, chez ces sujets, la production de ROS (en anglais, reactive oxygen species) augmente de l'ordre de $200 \%$ par à rapport à la concentration basale.

Comme c'est le cas du TNF- $\alpha$, I'IL-6 diminue également la signalisation de l'insuline [64] et, par conséquent, contribue à l'insulinorésistance (figure 2).

\section{Interaction entre I'adiponectine, la leptine, I'IL-6 et le TNF- $\alpha$}

L'adiponectine exerce un effet insulinosensibilisant, anti-athérogénique et antiinflammatoire [65]. Le taux élevé de I'adiponectine est parallèle à l'insulino-sensibilité. L'adiponectine joue un rôle comme une hormone insulino-sensibilisante dans le foie et les muscles et, par conséquent, augmente l'oxydation des acides gras dans les muscles squelettiques [66, 67]. Bahia et al. [68] ont effectué une étude sur les sujets minces et obèses, atteints du syndrome métabolique. Ils ont établi une corrélation entre le taux diminué de I'adiponectine et celui élevé du CRP, du fibrinogène et de PAI-I. Meller et al. [69] ont établi que la concentration de l'adiponectine diminue pendant les grossesses compliquées par le diabète maternel. Une baisse de l'adiponectine chez les mères atteintes de diabète gestationnel peut être responsable, en partie, de l'hyperglycémie. Nous avons récemment démontré que les mères atteintes du diabète gestationnel ont un taux d'adiponectine significativement bas par rapport aux sujets témoins [7]. Le taux plasmatique de I'adiponectine a une corrélation inverse avec l'insulino-résistance, le taux de triglycérides et de récepteurs de TNF- $\alpha$ [70]. 
L'adiponectine augmente l'insulino-sensibilité mais son mécanisme d'action n'est pas encore élucidé [71] bien que les îlots pancréatiques expriment les récepteurs 1 et 2 de l'adiponectine [72]. Le TNF- $\alpha$ et l'adiponectine peuvent contrecarrer leur mécanisme d'action : ils exercent des effets opposés sur la signalisation de I'insuline : le TNF- $\alpha$ diminue et l'adiponectine augmente la phosphorylation des tyrosines kinases des récepteurs de l'insuline [73]. De plus, le taux élevé de TNF- $\alpha$ peut aboutir à une baisse de la synthèse de l'adiponectine, car le premier freine la synthèse du dernier [74]. Lihn et al. [75] constatent que le TNF- $\alpha$ et I'IL-6 diminue la synthèse et l'expression de l'ARNm de l'adiponectine.

La leptine induit la sécrétion du TNF- $\alpha$ et de II'L-6 ainsi que celle de I'IL-1 $\beta$ [13]. La leptine semble exercer un effet immunomodulateur qui augmente le risque de l'infection. II est intéressant de noter que les souris ob/ob qui ne synthétisent pas la leptine sont susceptibles aux infections car leur système immunitaire est déprimé. Ces souris sont résistantes pour des pathologies auto-immunes qui nécessitent I'activation prolongée du système immunitaire. L'administration de la leptine exogène chez ces animaux restaure l'installation des pathologies auto-immunes. De la même manière, l'administration de la leptine chez la souris NOD (en anglais, non-obese diabetic) accélère l'installation du diabète [76]. Toutes ces observations sont en accord du fait que la leptine est l'inducteur de la différenciation cellulaire Th0 en phénotype Th1 qui sécrète les cytokines proinflammatoires qui sont impliquées dans l'installation des pathologies auto-immunes (voir le paragraphe précédent). II est à souligner que les femmes ont un taux élevé de leptine par à rapport aux hommes et qu'elles développent les maladies auto-immunes beaucoup plus fréquemment qu'eux. La leptine induit également le stress oxydatif et l'inflammation dans les cellules endothéliales [76]. La leptine induit I'activation de NF-kB [13]. Dans le diabète gestationnel, le rôle de la leptine n'est pas très clair mais nous avons récemment démontré que le taux de leptine et des cytokines pro-inflammatoires augmente chez ces sujets [7]. Lorsqu'on provoque une inflammation hépatique en injectant la concavaline-A chez la souris, on constate que cette situation est marquée par une augmentation de la sécrétion de la leptine et du TNF- $\alpha$ mais la concentration de l'adiponectine diminue chez ces animaux [15]. De plus, I'administration des anticorps anti-TNF- $\alpha$ diminue l'inflammation et restaure le taux de l'adiponectine. Ces résultats suggèrent que la leptine est pro-inflammatoire et que I'adiponectine exerce un effet antiinflammatoire.

\section{Effets anti-inflammatoires de I'insuline}

Nous avons mentionné ci-dessus que I'insulino-résistance favorise l'inflammation. Des études in vitro et in vivo ont démontré que I'insuline exerce un effet anti-inflammatoire à l'échelle cellulaire et moléculaire. L'insuline diminue la production de ROS à partir de cellules mononucléaires et la translocation de NF-kB vers le noyau [77]. De plus, I'insuline diminue la concentration de MCP-1, PAI-1, EGR-1 [78]. L'administration in vivo de l'insuline diminue non seulement la sévérité du diabète de type 2 mais provoque également une baisse significative de taux de MCP-1 et de CRP, un indicateur de l'état inflammatoire des patients [79].

On peut citer l'athérosclérose, qui est marquée par une corrélation entre l'inflammation et le métabolisme des lipides. II a été suggéré que cette pathologie est également provoquée en partie par des substances pro-inflammatoires [80]. L'athérosclérose est caractérisée par l'accumulation des monocytes et des macrophages au niveau des plaques d'athérome. L'augmentation des médiateurs proinflammatoires tels que le CRP et I'IL-6 augmente les risques de complications liées à l'athérosclérose. Ainsi, on peut dire que l'inflammation joue un rôle non seulement dans l'insulino-résistance mais également dans I'athérosclérose. L'insuline exerce ainsi un effet anti-inflammmatoire et anti-athérosclérotique. De ce fait, les substances qui augmentent la sensibilité de I'insuline tels que les thiazolidinediones (TZD) sont des agents antiinflammatoires et anti-athérogéniques [81]. Les TZD et l'insuline diminuent la production de ROS, l'expression de NADPH oxydase et la liaison de NF-kB dans les fractions nucléaires. De plus, les TZD diminuent la concentration circulante du TNF- $\alpha$ et du CRP. Dans des études expérimentales, I'insuline diminue la production de I'IL-6, du TNF- $\alpha$ et de I'IL-1 $\beta$ et concomitamment augmente celle des cytokines antiinflammatoires telles que I'IL-4 et I'IL-10 [82].

\section{Acides gras polyinsaturés (AGPI) $n$ - 3 exercent des effets anti-inflammatoires}

Les AGPI n-6 et plus précisément l'acide arachidonique $(A A)$, sont des constituants majeurs des membranes plasmiques. L'AA est le précurseur des prostaglandines et des leucotriènes qui ont des effets pro-inflammatoires et proathérogènes. Rappelons que les dérivés eicosanoides des AGPI n- 6 telles que la prostacycline sont des agents adipogéniques et ils induisent la différenciation des préadipocytes. L'effet adipogénique de prostacycline passe par ses récepteurs IP-R, couplés positivement à l'adénylate cyclase, et aboutit à la production d'AMP cyclique et à l'activation de la voie dépendante de la protéine kinase $A$ qui stimule alors l'adipogénèse dans les préadipocytes.

Quant aux AGPI n-3, ils exercent des effets cardioprotecteurs, anti-inflammatoires et immunosuppresseurs [83]. Les études épidémiologiques ont montré une faible incidence des maladies inflammatoires chez les Esquimaux et chez les Japonais qui consomment beaucoup de produits de mer, particulièrement les poissons qui sont des sources importantes d'ACPI n-3 comme l'acide eicosapentaenoïque, EPA, et I'acide docosahexaenoïque, DHA [83]. Ces acides gras exercent également des effets curatifs dans les maladies liées à I'inflammation, telles que l'arthrite rhumatoïde [84], le psoriasis [85] et COPD (en anglais, chronic obstructive pulmonary disease) pulmonaire [86]. II est intéressant de noter qu'un régime enrichi en $E P A / D H A n^{\prime}$ entraîne pas d'obésité chez le rat [87] ; de même, un régime hyperlipidique donné au rat après sevrage et enrichi en acide $\alpha$-linolénique, précurseur de EPA et de DHA, empêche le développement excessif du tissu adipeux [88].

Les études in vivo ont été entreprises chez les animaux pour vérifier le rôle modulateur des AGPI n-3 sur le fonctionnement ex vivo des lymphocytes: ils agiraient en diminuant la capacité des cellules mononuclées du sang à sécréter des cytokines telles que : IL-1 $\alpha$, IL-1 $\beta$, IL-2, IL-6 et tumor necrosis factor (TNF)- $\alpha$ [83]. Hudert et al. [89] ont réalisé une étude sur les souris fat- 1 transgéniques qui transforment spontanément les AGPI n-6 en n-3. Ils ont démontré que ces animaux sont protégés contre l'installation de colite inflammatoire du fait qu'ils synthétisent abondamment les dérivés de DHA, tels que les résolvines, et que l'expression de médiateurs pro-inflammatoires (NF-kB, TNF- $\alpha$, iNOS et IL-1b) diminue chez ces animaux.

Huang et al. [90] ont montré qu'un régime alimentaire enrichi en huile de poisson se traduisait par une diminution de l'expression de $\mathrm{CMH}$ II sur les macrophages péritonéaux des souris. Wallace et al. [91] ont observé que les AGPI n-3 alimentaires inhibaient la production des cytokines de type Th1 (IL-2 et IFN- $\gamma$ ) dans les lymphocytes spléniques des souris $\mathrm{C} 57 \mathrm{BL} / 6$ avec une diminution concomitante de la quantité des ARNm correspondants, mais sans modifier les cytokines sécrétées par les cellules Th2. Avec le même modèle, Arrington et al. [92] ont constaté une diminution de l'expression d'IL-2, mais également une stimulation de celle d'IL-4, ce qui augmente le rapport IL-4/IL-2. Ceci est en faveur d'une réorientation de la balance de différenciation des cellules Th0 vers le phénotype Th2. Nous avons démontré, 
chez les rats obèses issus de mères diabétiques, qu'un régime riche en AGPI n-3 exerce des effets bénéfiques: ce régime diminue l'incidence de la macrosomie, corrige les anomalies du métabolisme lipidique [93] et restaure le statut antioxydant [94]. De plus, ce régime module l'activation du système immunitaire chez ces animaux en diminuant la différenciation de cellules T vers le phénotype Th1 et en favorisant la différenciation vers le phénotype Th2 [6]. Ce régime riche en acides gras n-3 restaure aussi la signalisation calcique qui est modifiée par les cellules $T$ des bébés obèses [95].

Plusieurs études ont démontré la spécificité du mécanisme d'action de ces acides gras en ce qui concerne leur intervention au niveau de la signalisation cellulaire au cours de l'activation cellulaire $T$ : ils diminuent l'activation de NF-kB [96], la phosphorylation de ERK1/ERK2 [97, 98], le $\mathrm{pH}$ intracellulaire et modulent l'activité de différentes isoformes de protéine kinase $C$ [99] et de RasGRP [100].

Rappelons que les AGPI n-3 sont des activateurs de PPAR $\alpha$ dont les agonistes augmentent la sensibilité à l'insuline [101]. On peut résumer que les AGPI n-3 vont exercer un effet bénéfique en modifiant la différenciation cellulaire $T$ vers le phénotype Th2, en diminuant la sécrétion des cytokines pro-inflammatoires (TNF- $\alpha$ et IL-6) et en augmentant insulino-sensibilité au cours du diabète de type 2 et de l'obésité.

\section{Conclusion}

Le taux élevé du TNF- $\alpha$ et de l'IL-6 semble jouer un rôle clé dans l'induction de l'insulinorésistance (figure 2), en interférant, en partie, avec les effets anti-inflammatoires de l'insuline $[1,2]$. Cette conclusion peut être soutenue par des études génétiques. Le polymorphisme du promoteur des gènes du TNF- $\alpha$ et de I'IL-6 serait lié à une baisse de tolérance au glucose chez les sujets obèses. Une mutation du gène de TNF- $\alpha$ (G-308A) est associée à l'augmentation du taux de TNF- $\alpha$ circulant et d'un facteur de risque de 1.8 pour l'installation du diabète. Une mutation au niveau du gène de I'IL- 6 (C-124G) augmente aussi le risque de I'insulino-résistance [102]. Dans une population méditerranéenne-caucasienne, une corrélation entre la séquence Asp358Ala/ polymorphisme de CA-répété de gène I'IL-6 et l'obésité a été récemment établie [103]. Fernandez-Real et al. [104] ont identifié quatre allèles ( $A 1, A 2$, A4 et $A 4)$ sur le gène du récepteur de TNFR-2. Ils ont démontré que l'allèle $A 4$ prédispose les sujets humains à l'obésité et à l'augmentation du taux de la leptine.

Finalement, on peut dire que les adipocytes secrètent les adipokines et les cytokines proinflammatoires. II existe une intercommunica- tion entre les cytokines pro-inflammatoires et les effets anti-inflammatoires de l'adiponectine et de l'insuline, et de la différenciation de cellules T vers le phénotype Th1/Th2 pendant I'insulino-résistance. Les AGPI n-3 et les agonistes des PPAR $\alpha$ et PPAR $\gamma$ peuvent moduler ces situations physiologiques, en partie, via leurs effets immunomodulateurs.

\section{RÉFÉRENCES}

1. DANDONAP, ALJADAA, CHAUDHURIA, MOHANTY P, GARG R. Metabolic syndrome : a comprehensive perspective based on interactions between obesity, diabetes, and inflammation. Circulation 2005 ; 111 : 1448-54.

2. DANDONA P, ALJADA A. bandyopadhyay A. Inflammation: the link between insulin resistance, obesity and diabetes. Trends Immunol $2004 ; 25: 4-7$.

3. SCHMIDT MI, DUNCAN BB, SHARRETT AR, et al. Markers of inflammation and prediction of diabetes mellitus in adults (atherosclerosis risk in communities study): a cohort study. Lancet $1999 ; 353: 1649-52$.

4. WOOD SC, DHARMA RAO T, FREY B. Multidose streptozotocin induction of diabetes in $B A L B / C B y$ mice induces a $T$ cell proliferation defect in thymocytes which is reversible by interleukin-4. Cell Immunol 1999 ; 192 : 1-12.

5. MÜLLER A, SCHOTT-OHLY P, DOHLE C, GLEICHMANN H. Differential regulation of Th1-type and Th2-type cytokine profiles in pancreatic islets of C57BL/6 and BALB/C mice by multiple low doses of streptozotocin. Immunobiol $2002 ; 205$ : 35-50.

6. KHAN NA, YESSOUFOU A, KIM M, HICHAMI A. N-3 fatty acids modulate Th1 and Th2 dichotomy in diabetic pregnancy and macrosomia. | Autoimmun 2006 ; 26 : 268-77.

7. ATEGBO JM, GRISSA O, YESSOUFOU A, et al. Modulation of adipokines and cytokines in gestational diabetes and macrosomia. / Clin Endocrinol Metab 2006 ; 91 : 4137-43.

8. VERWAERDE C, DELANOYE A, MACIA L, TAILLEUXA, WOLOWCZUKI. Influence of high-fat feeding on both naive and antigenexperienced T-cell immune response in D010.11 mice. Scand / Immunol 2006; 64 : 457-66.

9. LI Z, SOLOSKI MJ, DIEHL AM. Dietary factors alter hepatic innate immune system in mice with nonalcoholic fatty liver disease. Hepatology $2005 ; 42: 880-5$.

10. HALAAS JL, GAJIWALA KS, MAFFEI M, et al. Weight-reducing effects of the plasma protein encoded by the obese gene. Science 1995; $269: 543-6$.

11. PAPATHANASSOGLOU E, EL-HASCHIMI K, LI XC, MATARESE G, STROM T, MANTZOROS C. Leptin receptor expression and signaling in lymphocytes : kinetics during lymphocyte activation, role in lymphocyte survival, and response to high fat diet in mice. I Immunol 2006 ; $176:$ 7745-52.

12. PACIFICO L, DI RENZO L, ANANIA C, et al. Increased T-helper interferon-gammasecreting cells in obese children. Eur J Endocrinol $2006 ; 154: 691-7$.

13. MATTIOLI B, STRAFACE E, QUARANTAMG, GIORDANI L, VIORA M. Leptin promotes differentiation and survival of human dendritic cells and licenses them for Th1 priming. I Immunol $2005 ; 174: 6820-8$.

14. PALACIO A, LOPEZ M, PEREZ-BRAVO $F$, MONKEBERG F, SCHLESINGER L. Leptin levels are associated with immune response in malnourished infants. / Clin Endocrinol Metab $2002 ; 87$ : 3040-6.

15. MORRIS AM, SENNELLO JA, FAYAD RA, ECKEL RH， DINARELLO CA， FANTUZZI G. T cell-mediated hepatic inflammation modulates adiponectin levels in mice: role of tumor necrosis factor alpha. Metabolism 2006; 55 : 555-9.

16. VIARDOT A, GREY ST, MACKAY F, CHISHOLM D. Potential anti-inflammatory role of insulin via the preferential polarization of effector $T$ cells towards a T-helper 2 phenotype. Endocrinology 2006 ; [Epub ahead of print].

17. ISSEMANN I, GREEN S. Activation of a member of the steroid hormone receptor superfamily by peroxisome proliferators. Nature $1900 ; 347$ : 645-50.

18. WAHLI W. Peroxisome proliferator-activated receptors (PPARs) : from metabolic control to epidermal wound healing. Swiss Med Wkly 2002 ; 132 : 83-91.

19. BRAISSANT O, FOUFELLE $F$, SCOTTO $C$, DAUCA M. WAHLI W. Differential expression of peroxisome proliferator-activated receptors (PPARs): tissue distribution of PPAR-alpha, -beta, and -gamma in the adult rat. Endocrinology 1996 ; 137 : 354-66.

20. CUNARD R, RICOTEM, DICAMPLID, et al. Regulation of cytokine expression by ligands of peroxisome proliferator activated receptors. J Immunol $2002 ; 168$ : 2795-802.

21. LOVETT-RACKE AE, HUSSAIN RZ, et al. Peroxisome proliferator-activated receptor alpha agonists as therapy for autoimmune disease. J Immunol $2004 ; 172$ : 5790-8.

22. CHINETTI-GBAGUIDI G, FRUCHART IC, STAELS $B$. Therapeutical effects of PPAR agonists assessed by biomarker modulation. Biomarkers $2005 ; 10$ (Suppl 1) : S30-S36.

23. JONES DC, DING $X$, DAYNES RA. Nuclear receptor peroxisome proliferator-activated receptor alpha (PPARalpha) is expressed in resting murine lymphocytes. The PPARalpha in T and $B$ lymphocytes is both transactivation and transrepression competent. / Biol Chem 2002 ; $277: 6838-45$. 
24. MARX N, KEHRLE B, KOHLHAMMER K, et al. PPAR activators as antiinflammatory mediators in human $\mathrm{T}$ lymphocytes: implications for atherosclerosis and transplantation-associated arteriosclerosis. Circ Res 2002 ; 90 : 703-10.

25. CUNARD R, DICAMPLI D, ARCHER DC, et al. WY14,643, a PPAR alpha ligand, has profound effects on immune responses in vivo. J Immunol $2002 ; 169: 6806-12$.

26. MARUYAMA S, KATO K, KODAMA M, et al. Fenofibrate, a peroxisome proliferatoractivated receptor alpha activator, suppresses experimental autoimmune myocarditis by stimulating the interleukin-10 pathway in rats. J Atheroscler Thromb $2002 ; 9$ : 87-92.

27. YANG XY, WANG LH, CHEN T, et al. Activation of human T lymphocytes is inhibited by peroxisome proliferator-activated receptor gamma (PPARgamma) agonists. PPARgamma Co-association with transcription factor NFAT. J Biol Chem 2000 ; 275 : 4541-4.

28. KURATA H, LEE HJ, O'GARRA A, ARAI N. Ectopic expression of activated Stat6 induces the expression of Th2-specific cytokines and transcription factors in developing Th1 cells. Immunity $1999 ; 11: 677-88$.

29. YESSOUFOU A, HICHAMI A, BESNARD P, MOUTAIROU K, KHANNA. Peroxisome proliferator-activated receptor alpha deficiency increases the risk of maternal abortion and neonatal mortality in murine pregnancy with or without diabetes mellitus : Modulation of T cell differentiation. Endocrinology 2006; 147 : 4410-8.

30. CATALANO PM, NIZIELSKI SE, SHAO I, PRESTON L, QIAO L, FRIEDMAN JE. Downregulated IRS-1 and PPARgamma in obese women with gestational diabetes: relationship to FFA during pregnancy. Am / Physiol Endocrinol Metab $2002 ; 282$ : E522-E523.

31. KLIEWER SA, SUNDSETH SS, JONES SA, et al. Fatty acids and eicosanoids regulate gene expression through direct interactions with peroxisome proliferator-activated receptors alpha and gamma. Proc Natl Acad Sci USA 1997 ; 94 : 4318-23.

32. RISTOW M, MULLER-WIELAND D, PFEIFFER A, KRONE W, KAHN CR. Obesity associated with a mutation in a genetic regulator of adipocyte differentiation. N Engl / Med 1998; 339 : 953-9.

33. AUGSTEIN $P$, DUNGER A, HEINKE $P$, et al. Prevention of autoimmune diabetes in NOD mice by troglitazone is associated with modulation of ICAM-1 expression on pancreatic islet cells and IFN-gamma expression in splenic T cells. Biochem Biophys Res Commun 2003 ; 304 : 378 84.

34. BARAK Y, NELSON MC, ONG ES, et al. PPAR gamma is required for placental, cardiac, and adipose tissue development. Mol Cell $1999 ; 4$ : 585-95.
35. YOUSSEF J, BADR M. Role of Peroxisome Proliferator-Activated Receptors in inflammation Control. / Biomed Biotechnol 2004 ; 2004 : 156-66.

36. RICOTE M, LI AC, WILLSON TM, KELLY C], GLASS CK. The peroxisome proliferatoractivated receptor-gamma is a negative regulator of macrophage activation. Nature 1998 ; 391 : 79-82.

37. THIERINGER R, FENYK-MELODY JE, LE GRAND $C B$, et al. Activation of peroxisome proliferatoractivated receptor gamma does not inhibit IL-6 or TNF-alpha responses of macropages to lipopolysaccharide in vitro or in vivo. I Immunol $2000 ; 164$ : 1046-54.

38. CREELY SI, MCTERNAN PG, KUSMINSKI CM, et al. Lipopolysaccharide activates an innate immune system response in human adipose tissue in obesity and type 2 diabetes. Am J Physiol Endocrinol Metab 2006 ; [Epub ahead of print].

39. HOTAMISLIGIL GS, ARNER P, CARO IF, ATKINSON RL, SPIEGELMAN BM. Increased adipose expression of tumor necrosis factor- in human obesity and insulin resistance. I Clin Invest $1995 ; 95: 2409-15$.

40. HOTAMISLIGIL GS, BUDAVARI A, MURRAY D, SPIEGELMAN BM. Reduced tyrosine kinase activity of the insulin receptor in obesitydiabetes. Central role of tumor necrosis factor/ Clin Invest 1994 ; 94 : 1543-9.

42. HOTAMISLIGIL GS, MURRAY DL, CHOY LN, SPIEGELMAN BM. Tumor necrosis factor alpha inhibits signaling from the insulin receptor. Proc Natl Acad Sci USA 1994 ; 91 : 4854-8.

41. SARTIPY P, LOSKUTOFF DJ. Monocyte chemoattractant protein 1 in obesity and insulin resistance. Proc Natl Acad Sci USA 2003; 100 : 7265-70.

43. KERN PA, SAGHIZADEH M, ONG JM, BOSCH RJ, DEEM R, SIMSOLO RB. The expression of tumor necrosis factor in human adipose tissue. Regulation by obesity, weight loss, and relationship to lipoprotein lipase. I Clin Invest $1995 ; 95: 2111-9$.

44. WEISBERG SP, MCCANN D, DESAI M, ROSENBAUM M, LEIBEL RL, FERRANTE JR. AW. Obesity is associated with macrophage accumulation in adipose tissue. / Clin Invest $2003 ; 112$ : 1796-808.

45. LACASA D, TALEB S, KEOPHIPHATH M, MIRANVILLE A, CLEMENT K. Macrophages secreted factors impair human adipogenesis : involvement of pro-inflammatory state in preadipocytes. Endocrinology 2006 ; [Epub ahead of print].

46. WINKLER G, CSEH K, BARANYI E, et al. Tumour necrosis factor system in insulin resistance in gestational diabetes. Diabetes Res Clin Pract $2002 ; 56: 93-9$.
47. CLEMENT K, VIGUERIE N, POITOU C, et al. Weight loss regulates inflammation-related genes in white adipose tissue of obese subjects. FASEB J $2004 ; 18$ : 1657-69.

48. KOPP HP, KOPP CW, FESTA A, et al. Impact of weight loss on inflammatory proteins and their association with the insulin resistance syndrome in morbidly obese patients. Arterioscler Thromb Vasc Biol 2003 ; 23 : 1042-7.

49. ESPOSITO K, PONTILLO A, DI PALO C, et al. Effect of weight loss and lifestyle changes on vascular inflammatory markers in obese women : a randomized trial. JAMA $2003 ; 289$ : 1799-804.

50. DANDONA P, WEINSTOCK R, THUSU K, ABDEL-RAHMAN E, ALJADA A, WADDEN T. Tumor Necrosis Factor- in Sera of Obese Patients : Fall with Weight Loss. / Clin Endocrinol Metab $1998 ; 83: 2907-10$.

51. CHEATHAM B, KAHN CR. Insulin action and insulin signalling network. Endocr Rev 1995 ; $16: 117-42$.

52. ECKEL RH. Insulin resistance : an adaption for weight maintenance. Lancet 1992; 340 : 1452-3.

53. HOTAMISLIGIL GS, SHARGILL NS, SPIEGELMAN BM. Adipose expression of tumor necrosis factor- : direct role in obesity-linked insulin resistance. Science 1993 ; 259 : 87-91.

54. SAMAD F, UYSAL KT, WIESBROCK SM, PANDEY M, HOTAMISLIGIL GS, LOSKUTOFF D). Tumor necrosis factor a is a key component in the obesity-linked elevation of plasminogen activator inhibitor I. Proc Natl Acad Sci USA $1999 ; 96: 66902-7$

55. MOHAMED-ALI V, GOODRICK S, RAWESH A, et al. Subcutaneous adipose tissue releases interleukin- 6 but not tumour necrosis factor- $\alpha$, in vivo. / Clin Endocrinol Metab 1997; 82 : 4196-200.

56. SCHOBITZ B, DE KLOET ER, SUTANTO W, HOLSBOER F. Cellular localization of interleukin 6 mRNA and interleukin 6 receptor mRNA in rat brain. Eur / Neurosci 1993 ; 5 : 1426-35.

57. WALLENIUS K, WALLENIUS V, SUNTER D, DICKSON SL, JANSSON JO. Intracerebroventricular interleukin-6 treatment decreases body fat in rats. Biochem Biophys Res Commun 2002 ; $293: 560-5$.

58. ETTINGER Jr. WH, SUN WH, BINKLEY N, KOUBAE, ERSHLER W. Interleukin-6 causes hypocholesterolemia in middle-aged and old rhesus monkeys. I Gerontol A Biol Sci Med Sci 1995 ; 50 : M137-M140.

59. BASTARD JP, MAACHI M, VAN NHIEU JT, et al. Adipose tissue IL-6 content correlates with resistance to insulin activation of glucose uptake both in vivo and in vitro. J Clin Endocrinol Metab 2000 ; 87 : 2084-9. 
60. PICKUP JC, MATTOCK MB, CHUSNEY GD BART D. NIDDM as a disease of the innate immune system association of acute-phase reactants and interleukin- 6 with metabolic syndrome X. Diabetologia 1997 ; 40 : 1286-92.

61. MULLER S, MARTIN S, KOENIG W, et al. Impaired glucose tolerance is associated with increased serum concentrations of interleukin 6 and co-regulated acute-phase proteins but not TNF-alpha or its receptors. Diabetologia 2002 ; $45: 805-12$.

62. STERNBERG EM. The stress response and the regulation of inflammatory disease. Ann Intern Med $1992 ; 117: 854-66$.

63. MOHANTY $P$, HAMOUDA W, GARG R, ALJADA A, GHANIM H, DANDONA P. Glucose challenge stimulates reactive oxygen species (ROS) generation by leucocytes. I Clin Endocrinol Metab $2000 ; 85$ : 2970-3.

64. SENN J), et al. Interleukin-6 induces cellular insulin resistance in hepatocytes. Diabetes $2002 ; 51: 3391-9$

65. DIEZ J), IGLESIAS P. The role of the novel adipocyte-derived hormone adiponectin in human and possible biological roles. Eur J Endocrinol $2003 ; 148: 293-300$.

66. COMBS TP, BERG AH, OBICI S, SCHERER PE, ROSSETTI L. Endogenous glucose production is inhibited by the adipose-derived protein Acrp30. J Clin Invest $2001 ; 108$ : 1875-81.

67. HOTTA K, FUNAHASHIT, BODKIN NL, et al. Circulating concentrations of the adipocyte protein adiponectin are decreased in paralle with reduced insulin sensibility during the progression of type 2 diabetes in Rhesus monkeys. Diabetes 2001 ; 50 : 1126-33.

68. BAHIA L, AGUIAR LG, VILLELA N, et al. Relationship between adipokines, inflammation, and vascular reactivity in lean controls and obese subjects with metabolic syndrome. Clinics 2006 ; $61: 433-40$.

69. MELLER M, QIU C, VADACHKORIA S, ABETEW DF, LUTHY DA, WILLIAMS MA. Changes in Placental Adipocytokine Gene Expression Associated with Gestational Diabetes MellitusA. Physiol Res 2006 ; 55 : 501-12.

70. FERNANDEZ-REAL JM, LOPEZ-BERMEJO A, CASAMITJANA R, RICART W. Novel interactions of adiponectin with the endocrine system and inflammatory parameters. / Clin Endocrinol Metab $2000 ; 88$ : 2714-8.

71. ARITA Y, KIHARA S, OUCHI N, et al. Paradoxical decrease of an adipose-specific protein, adiponectin, in obesity. Biochem Biophys Res Commun $1999 ; 257: 79-83$
72. STAIGER K, STEFAN N, STAIGER H, et al. Adiponectin is functionally active in human islets but does not affect insulin secretory function or beta-cell lipoapoptosis. I Clin Endocrinol Metab $2005 ; 90: 6707-13$.

73. STEFAN N, VOZAROVA B, FUNAHASHI T, et al. Plasma adiponectin concentration is associated with skeletal muscle insulin receptor tyrosine phosphorylation, and low plasma concentration precedes a decrease in whole-body insulin sensitivity in humans. Diabetes $2002 ; 51$ : 1884-8.

74. RUAN H, LODISH HF. Regulation of insulin sensitivity by adipose tissue-derived hormones and inflammatory cytokines. Curr Opin Lipidol 2004 ; 15 : 297-302.

75. LIHN AS, RICHELSEN B, PEDERSEN SB, et al. Increased expression of TNF-alpha, IL-6, and IL-8 in HALS : implications for reduced adiponectin expression and plasma levels. Am / Physiol Endocrinol Metab 2003; 285: E1072E1080.

76. MATARESE G, LA CAVA A, SANNA V, et al. Balancing susceptibility to infection and autoimmunity : a role for leptin? Trends Immunol 2002 ; 23 : 182-7.

77. DANDONA $P$, ALJADA A, MOHANTY $P$, GHANIM H, HAMOUDA W, ASSIAN E, AHMAD $S$. Insulin inhibits intranuclear nuclear factor kappaB and stimulates IkappaB in mononuclear cells in obese subjects : evidence for an anti-inflammatory effect? J Clin Endocrinol Metab. $200 ; 86: 3257-65$.

78. HANSEN TK, THIEL S, KNUDSEN ST, et al. Elevated levels of mannan-binding lectin in patients with type 1 diabetes. / Clin Endocrinol Metab 2003 ; 88 : 4857-61.

79. TAKEBAYASHI K, ASO Y, INUKAI T. Initiation of insulin therapy reduces serum concentrations of high-sensitivity $\mathrm{C}$-reactive protein in patients with type 2 diabetes. Metabolism 2004 ; 53 : 693-9.

80. ROSS R. Atherosclerosis-an inflammatory disease. N Engl / Med 1999 ; 340 : 115-26.

81. GHANIM H, GARG R, ALJADA A, et al. suppression of nuclear factor- $\mathrm{K} B$ and stimulation of inhibitor $\kappa B$ by troglitazone : evidence for an anti-inflammatory effect and a potential antiatherosclerotic effect in the obese. / Clin Endocrinol Metab 2001 ; 86 : 1306-12.

82. JESCHKE MG, KLEIN D, BOLDER U, EINSPANIER R. Insulin attenuates the systemic inflammatory response in endotoxemic rats. Endocrinology $2004 ; 145$ : 4084-93.
83. CALDER PC. n-3 polyunsaturated fatty acids, inflammation, and inflammatory diseases. Am J Clin Nutr 2006 ; 83 : 1505S-1519S.

84. KREMER JM. n-3 fatty acid supplements in rheumatoid arthritis. Am / Clin Nutr 2000 ; 71 : 349S-351S

85. MORI TA, BEILIN LJ. Omega-3 fatty acids and inflammation. Curr Atheroscler Rep 2004; 6 : 461-7.

86. MATSUYAMA W, MITSUYAMA H, WATANABE $M$, et al. Effects of omega-3 polyunsaturated fatty acids on inflammatory markers in COPD. Chest $2005 ; 128: 3817-27$.

87. PARRISH CC, PATHY DA, ANGEL A. Dietary fish oils limit adipose tissue hypertrophy in rats. Metabolism $1990 ; 39$ : 217-9.

88. RACLOT T, GROSCOLAS R, LANGIN D, FERRÉ $P$. Site-specific regulation of gene expression by n-3 polyunsaturated fatty acids in rat white adipose tissues. / Lipid Res 1997 ; 38 : 1963-72.

89. HUDERT CA, WEYLANDT KH, LU Y, et al. Transgenic mice rich in endogenous omega-3 fatty acids are protected from colitis. Proc Natl Acad Sci USA 2006 ; 103 : 11276-81.

90. HUANG SC, MISFELDT ML, FRITSCHE KL. Dietary fat influences la antigen expression and immune cell populations in the murine peritoneum and spleen. / Nut $1992 ; 122$ : 1219-31.

91. WALLACE FA, MILES EA, EVANS C, STOCK TE, YAQOOB P, CALDER PC. Dietary fatty acids influence the production of Th1 - but not Th2type cytokines. J Leukoc Biol 2001 ; 69 : 449-57.

92. ARRINGTON JL, CHAPKIN RS, SWITZER KC, MORRIS JS, MCMURRAY DN. Dietary n-3 polyunsaturated fatty acids modulate purified murine T-cell subset activation. Clin Exp Immunol 2001 ; 125 : 499-507.

93. SOULIMANE-MOKHTARI NA, GUERMOUCHE $B$, YESSOUFOU A, et al. Modulation of lipid metabolism by $n-3$ polyunsaturated fatty acids in gestational diabetic rats and their macrosomic offspring. Clin Sci (Lond) 2005 ; 109 : 28795.

94. YESSOUFOU A, SOULAIMANN N, MERZOUK SA, et al. N-3 fatty acids modulate antioxidant status in diabetic rats and their macrosomic offspring. Int J Obes (Lond) 2006 ; 30 : 739-50.

95. GUERMOUCHE B, YESSOUFOU A, SOULIMANE $\mathrm{N}$, et al. $\mathrm{n}-3$ fatty acids modulate $\mathrm{T}$-cell calcium signaling in obese macrosomic rats. Obes Res $2004 ; 12: 1744-53$

96. DENYS A, HICHAMI A, KHAN NA. n-3 PUFAs modulate T-cell activation via protein kinase C-alpha and -epsilon and the NF-kappaB signaling pathway. J Lipid Res 2005 ; 46 : 752-8. 
97. DENYSA, AIRES V, HICHAMI A, KHAN NA. Thapsigargin-stimulated MAP kinase phosphorylation via CRAC channels and PLD activation inhibitory action of docosahexaenoic acid. FEBS Lett $2004 ; 564$ : 177-82.

98. DENYS A, HICHAMI A, KHAN NA. Eicosapentaenoic acid and docosahexaenoic acid modulate MAP kinase (ERK1/ERK2) signaling in human T cells. J Lipid Res 2001 ; 42 : 2015-20.

99. MADANIS, HICHAMI A, LEGRAND A, BELLEVILLE J, KHAN NA. Implication of acyl chain of diacylglycerols in activation of different isoforms of protein kinase C. FASEB / $2001 ; 15$ : 2595-601.
100. MADANI S, HICHAMI A, CHERKAOUI-MALK M, KHAN NA. Diacylglycerols containing Omega 3 and Omega 6 fatty acids bind to RasGRP and modulate MAP kinase activation. J Biol Chem 2004 ; 279 : 1176-83.

101. BLANQUART C, MANSOURI R, PAUMELLE R, FRUCHART JC, STAELS B, GLINEUR C. The protein kinase $C$ signaling pathway regulates a molecular switch between transactivation and transrepression activity of the peroxisome proliferator-activated receptor alpha. Mol Endocrinol $2004 ; 18$ : 1906-18.

102. KUBASZEK A, PIHLAJAMAKI J, KOMAROVSKI V, et al. Finnish Diabetes Prevention Study. Promoter polymorphisms of the TNF- $\alpha(\mathrm{G}-308 \mathrm{~A})$ and IL-6 (C-174G) genes predict the conversion from impaired glucose tolerance to type 2 diabetes: the Finnish diabetes prevention study. Diabetes 2003 ; 52 : 1872-6.

103. ESTEVE E, VILLUENDAS G, MALLOLAS J, et al. Polymorphisms in the interleukin-6 receptor gene are associated with body mass index and with characteristics of the metabolic syndrome. Clin Endocrinol (Oxf) 2006 ; 65 : 88-91 ; (Oxf).

104. FERNANDEZ-REAL JM, VENDRELL J, RICART W, et al. Polymorphism of the tumor necrosis factor-alpha receptor 2 gene is associated with obesity, leptin levels, and insulin resistance in young subjects and diet-treated type 2 diabetic patients. Diabetes Care 2000 ; 23 : 831-7. 